www.nature.com/jhg

\title{
Molecular analysis of potassium ion channel genes in sudden death cases among patients administered psychotropic drug therapy: are polymorphisms in LQT genes a potential risk factor?
}

\author{
Sayako Kamei ${ }^{1,2}$, Noriko Sato ${ }^{1}$, Yuta Harayama ${ }^{1}$, Miyako Nunotani ${ }^{1}$, Kanae Takatsu ${ }^{1}$, Tetsuya Shiozaki ${ }^{1}$, \\ Tokutaro Hayashi ${ }^{1}$ and Hideki Asamura ${ }^{1}$
}

Psychotropic drugs can pose the risk of acquired long QT syndrome (LQTS). Unexpected autopsy-negative sudden death in patients taking psychotropic drugs may be associated with prolonged QT intervals and life-threatening arrhythmias. We analyzed genes that encode for cardiac ion channels and potentially associated with LQTS, examining specifically the potassium channel genes KCNQ1 and KCNH2 in 10 cases of sudden death involving patients administered psychotropic medication in which autopsy findings identified no clear cause of death. We amplified and sequenced all exons of KCNQ1 and KCNH2, identifying G643S, missense polymorphism in KCNQ1, in 6 of the 10 cases. A study analysis indicated that only $11 \%$ of 381 healthy Japanese individuals carry this polymorphism. Reports of previous functional analyses indicate that the G643S polymorphism in the KCNQ1 potassium channel protein causes mild $I_{\mathrm{Ks}}$ channel dysfunction. Our present study suggests that administering psychotropic drug therapy to individuals carrying the G643S polymorphism may heighten the risk of prolonged QT intervals and life-threatening arrhythmias. Thus, screening for the G643S polymorphism before prescribing psychotropic drugs may help reduce the risk of unexpected sudden death. Journal of Human Genetics (2014) 59, 95-99; doi:10.1038/jhg.2013.125; published online 28 November 2013

Keywords: KCNH2; KCNQ1; long QT syndrome; psychotropic drugs; sudden death

\section{INTRODUCTION}

In some cases, forensic pathologists called on to examine the case of sudden and unexpected death in patients administered medication to treat psychological disorders are unable to identify any clear cause of death, a conclusion that suggests a functional cause of death. Papers report significantly higher mortality rates in psychiatric patients than among the general population. ${ }^{1-3}$ This high rate may be linked to psychotropic medication. ${ }^{3}$ Some psychotropic drugs affect the ion channels that control cardiac action..$^{4-10}$ The Arizona Center for Education and Research on Therapeutics has published lists of drugs, including some of antipsychotic or antidepressant drugs, associated with prolonged QT intervals. ${ }^{11}$ On the basis of an analysis of electrocardiograms for 1017 patients suffering from schizophrenia, Ozeki et al. ${ }^{12}$ reported an increased risk of prolonged QT intervals with chlorpromazine, levomepromazine and haloperidol. Druginduced long QT syndrome (LQTS) is known as acquired LQTS. Prolonged QT intervals can increase the risk of episodes of torsades de pointes, potentially resulting in ventricular fibrillations of lifethreatening arrhythmias. Congenital LQTS is another type of LQTS caused by polymorphisms in the genes responsible for prolonged QT intervals. Studies to date have described 13 genes (LQT1 to LQT13) associated with congenital LQTS. ${ }^{13}$ Silvia et al. ${ }^{14}$ report that carriers of the certain polymorphisms in LQT genes were at risk of developing torsade de pointes unexpectedly if exposed to cardiac or non-cardiac drugs that block potassium channels. More than 600 polymorphisms and splice site altering polymorphisms have been identified in LQT genes; $\sim 75 \%$ of clinically definite LQTS are caused by polymorphisms in 3 genes: LQT1, LQT2 and LQT3. ${ }^{15}$

In this study, based on the hypothesis that individuals carrying certain polymorphisms in the LQT genes may be at risk of lifethreatening arrhythmias if exposed to psychotropic drugs that block cardiac ion channels, we looked for major congenital LQTS-causing genes (KCNQ1 and KCNH2) in 10 cases of autopsy-negative sudden death involving patients administered psychotropic medication for genetic variations affecting the potassium.

\section{MATERIALS AND METHODS}

Materials

This study was approved by the Ethics Committee of Shinshu University. We analyzed 10 samples from autopsy-negative Japanese patients who had suffered

${ }^{1}$ Department of Legal Medicine, Shinshu University School of Medicine, Nagano, Japan and ${ }^{2}$ Department of Biochemistry, Graduate School of Oral Medicine, Matsumoto Dental University, Nagano, Japan

Correspondence: Professor H Asamura, Department of Legal Medicine, Shinshu University School of Medicine, Asahi 3-1-1, Matsumoto, Nagano 390-8621, Japan. E-mail: asamura@shinshu-u.ac.jp

Received 24 July 2013; revised 5 November 2013; accepted 6 November 2013; published online 28 November 2013 
sudden and unexpected death during medication therapy for psychological disorders (the psychotropic drug group). All patients were free of previous diagnosed medical conditions other than psychological disorders and had no family history of sudden death. Table 1 provides information for the
10 patients. Our study excluded patients whose blood concentrations of psychotropic drugs were beyond the therapeutic ranges for drug therapy. We extracted DNA from blood samples using the QIAamp DNA Blood Mini Kit (Qiagen, Hilden, Germany) in accordance with the specified protocol.

Table 1 Case profiles for psychoactive drug group

\begin{tabular}{|c|c|c|c|c|}
\hline Case no. & Age & Sex & $\begin{array}{l}\text { Psychiatric } \\
\text { diagnosis }\end{array}$ & Prescription drugs \\
\hline 1 & 20 s & Female & Schizophrenia & Risperidon ${ }^{a}$, Paroxetine ${ }^{\mathrm{a}}$, Etizolam, Levomepromazine ${ }^{\mathrm{b}}$, Promethazine ${ }^{\mathrm{a}}$, Flunitrazepam, Lorazepam \\
\hline 2 & $40 \mathrm{~s}$ & Female & Schizophrenia & Risperidon ${ }^{\mathrm{a}}$ Trazodone ${ }^{\mathrm{a}}$, Diazepam, Etizolam, Brotizolam \\
\hline 3 & $60 \mathrm{~s}$ & Male & Schizophrenia & Chorpromazine $^{a}$, Levomepromazine ${ }^{b}$, Nitrazepam, Carbamazepine, Phenobarbital, Promethazine ${ }^{a}$ \\
\hline 4 & $20 \mathrm{~s}$ & Male & Schizophrenia & Risperidon ${ }^{a}$, Diazepam, Flunitrazepam, Cloxazolam, Brotizolam \\
\hline 5 & $30 \mathrm{~s}$ & Female & Schizophrenia & Haloperidol $^{\mathrm{a}}$, Risperidon ${ }^{\mathrm{a}}$, Diazepam, Quetiapine ${ }^{\mathrm{a}}$ \\
\hline 6 & $50 \mathrm{~s}$ & Female & Anxiety neurosis & Etizolam, Zolpidem \\
\hline 7 & 20 s & Male & Schizophrenia & Risperidon ${ }^{a}$, Olanzapine ${ }^{a}$, Alprazolam, Diazepam, Flunitrazepam \\
\hline 8 & $30 \mathrm{~s}$ & Male & Schizophrenia & Chorpromazine $^{a}$, Risperidon ${ }^{a}$, Olanzapine ${ }^{a}$, Duloxetine, Phenobarbital, Flunitrazepam, Promethazine ${ }^{a}$ \\
\hline 9 & 30 s & Male & Schizophrenia & $\begin{array}{l}\text { Haloperidola }{ }^{a} \text { Chorpromazine }{ }^{a} \text {, Risperidon }{ }^{a} \text {, Quetiapine }{ }^{a} \text {, Estazolam, Nitrazepam, } \\
\text { Flunitrazepam, Brotizolam, Amobarbital }\end{array}$ \\
\hline 10 & $40 \mathrm{~s}$ & Female & Schizophrenia & Quetiapine $^{a}$, Carbamazepine, Levomepromazine ${ }^{\mathrm{b}}$, Estazolam, Lorazepam, Etizolam \\
\hline
\end{tabular}

aDrugs that can potentially prolong QT interval (http://www.crediblemeds.org/).

${ }^{b}$ Ozeki et al. ${ }^{12}$ reports that levomepromazine can potentially prolong QT interval.

Table 2 Each primer sequence and PCR conditions

\begin{tabular}{|c|c|c|c|c|c|c|c|}
\hline \multirow[b]{2}{*}{ Gene } & \multicolumn{2}{|c|}{ Primer } & \multirow{2}{*}{$\begin{array}{c}\text { Primer } \\
\text { concentration } \\
(\mu M)\end{array}$} & \multirow[b]{2}{*}{$\begin{array}{l}\text { Amplicon } \\
\text { size (bp) }\end{array}$} & \multirow[b]{2}{*}{$\begin{array}{c}\mathrm{MgCl}_{2} \\
(\mathrm{mM})\end{array}$} & \multirow[b]{2}{*}{$\operatorname{Tm}\left({ }^{\circ} \mathrm{C}\right)$} & \multirow[b]{2}{*}{ References } \\
\hline & Forward $\left(5^{\prime}-3^{\prime}\right)$ & Reversed $\left(5^{\prime}-3^{\prime}\right)$ & & & & & \\
\hline \multicolumn{8}{|c|}{$K C N Q 1(L Q T 1)$} \\
\hline Exon 1.1 & CTCGCCTTCGCTGCAGCTC & GCGCGGGTCTAGGCTCACC & 0.3 & 334 & 1.5 & TD 70-60 & 16 \\
\hline 1.2 & CGCCGCGGCCCCAGTTGC & CAGAGCTCCCCCACACCAG & 0.3 & 244 & 1.5 & TD 70-60 & 16 \\
\hline 2 & AATGGATGACTGGGTTTTCG & TATCAGGGCAGGACCAATGT & 0.3 & 363 & 1.5 & 60 & \\
\hline 3 & TTCTCAGGGTGTCCTTCAGC & AGGGGACTCCATCTGGTAGG & 0.3 & 334 & 1.5 & 60 & \\
\hline 4 & ATCCGAGGTGTCTCCATGTC & CATCTGAGCAAGGTGGATGG & 0.3 & 326 & 1.5 & 60 & \\
\hline 5 & AGGGACACCCATGCCATC & CTGCTCCCTCCGTCCTGT & 0.3 & 246 & 1.5 & 65 & \\
\hline 6 & CTTAGGCGTCTGCACAGGAG & GCACAGGTTTGTGGACAGAG & 0.3 & 359 & 1.5 & 65 & \\
\hline 7 & GCTCTGTTCCTGGTGCTTTC & CGTAAGTGGGTCTGCTCACA & 0.3 & 367 & 1.5 & 60 & \\
\hline 8 & ATACCTGGCCTTCCCACAAC & AAGCAGAGTATGCCCCACAG & 0.3 & 333 & 1.5 & 60 & \\
\hline 9 & TCAAGCCTGTGACTCTGAGGT & TGACACAGGCTGTACCAAGC & 0.3 & 388 & 1.5 & 65 & \\
\hline 10 & GCTGCACAGGCACTCTGG & GAAGCTCCACCCTCTGTCTG & 0.3 & 364 & 1.5 & 60 & \\
\hline 11 & ACTGATTGTCAGGGCTGGAG & TGGGCACTAGGCGAGTAGAT & 0.3 & 376 & 1.5 & 65 & \\
\hline 12 & TCTGGAAGGATCCAGTCTGC & GCCTCCAAGGATAGGTCCTC & 0.3 & 392 & 1.5 & 60 & \\
\hline 13 & AACCAGGCTTATGCCATCAC & GGTGGTTGAGAGGCAAGAAC & 0.3 & 362 & 1.5 & 60 & \\
\hline 14 & GTCAAGCTGTCTGTCCCACA & CCCTGGCTTTCATTTCATGT & 0.3 & 270 & 1.5 & 60 & \\
\hline 15 & ACCGTACCACCCCTGGTATT & CCCCTCTTGGGAGTTGCT & 0.3 & 393 & 1.5 & 60 & \\
\hline 16 & GTTGGCACCTTCCCTTCTCT & АСTCTTGGCCTCCССTCTC & 0.3 & 391 & 1.5 & 60 & \\
\hline \multicolumn{8}{|c|}{ KCNH2 (LQT2) } \\
\hline Exon 1 & CCGCCCATGGGCTCAGG & CATCCACACTCGGAAGAAGT & 0.3 & 144 & 1.5 & TD 62-50 & 16,17 \\
\hline 2 & CGCАСTCTCCTCACCGCCCC & CCСTCTTGACCCCGCCCСTG & 0.3 & 305 & 1.5 & TD 65-70 & 17 \\
\hline 3 & GTTCACTTCCCACCTCCAAA & CATCCTGCGTGGCTTTCT & 0.3 & 378 & 1.5 & 60 & \\
\hline 4.1 & ACGACCACGTGCCTCTCCTCTC & GGGACCCACCAGCGCACGCCG & 0.3 & 267 & 1.5 & 64.2 & 17,18 \\
\hline 4.2 & CCCTGGACGAAGTGACAGCCATGG & GGCTGGGGCGGAACGGGTCC & 0.3 & 319 & 1.5 & 67.7 & 18 \\
\hline 5 & CCTCCAAGGTGAGAGGAGA & GCCTGACCACGCTGCCTCT & 0.3 & 279 & 1.5 & 60 & \\
\hline 6 & TCСТСТСССТАСАССАССТG & CCTGCTGCTGGTCATCTACA & 0.3 & 354 & 1.5 & 62 & \\
\hline 7 & AAGTTCCAGGGCCTCACTCT & TCTTGCTCATGTGCACCTTT & 0.3 & 398 & 1.5 & 62 & \\
\hline 8 & CTCTGCCACCCCACTCTTC & GTCCCTGCAGAGGCTGAC & 0.3 & 300 & 1.5 & 64 & \\
\hline 9.1 & TTCCTCTACTGCCCAGGCTA & CACCGCCCTGTACTTCATCT & 0.3 & 378 & 1.5 & 62 & \\
\hline 9.2 & AGAAGGCTCGCACCTCTTG & GGTGGGATGGTGGAGTAGAG & 0.3 & 397 & 1.5 & 62 & \\
\hline 10 & ACAGCTGGAAGCAGGAGGAT & GGCTGAGCTCCCTGTCCT & 0.3 & 275 & 1.5 & 70 & \\
\hline 11 & AAGGGATGGGAAGGTCTGAG & CACTGAAAGGGCCCTGATAC & 0.3 & 247 & 1.5 & TD 58-70 & \\
\hline 12 & CTGCAGCCAGAGAGCAGAG & CTCTGTTTCCCACAGACACG & 0.3 & 379 & 0.75 & 50 & \\
\hline 13 & GСССТСТСССТСТАССАGAC & AGAAGAGCAGCGACACTTGC & 0.3 & 395 & 1.5 & 65 & \\
\hline 14 & GTCACGGTACATCGAGGAAG & CCCACTTCTCTGAGCATCC & 0.3 & 250 & 1.5 & 62 & \\
\hline 15 & СTCСTССTCСАTGGССTCT & CCTTGATCCCTGGGTGAG & 0.3 & 243 & 1.5 & 64 & \\
\hline
\end{tabular}

Abbreviation: TD, touch-down. 
After obtaining informed consent, we also extracted DNA from 100 Japanese non-LQTS individuals as negative controls (the control group).

\section{PCR and sequencing analysis}

All exons of the two genes, KCNQ1 (LQT1) and KCNH2 (LQT2), were generated using newly designed primer sets, except for exon 1 of LQT1 and exons 1, 2, 4 and 11 of LQT2. ${ }^{16-18}$ For exon 1 of LQT1 and exons 1, 2 and 11 of LQT2, we performed touch-down PCR techniques ${ }^{19}$ in addition to mixing Q-Solution (Qiagen) into the PCR solution. Table 2 shows each primer sequence and the PCR conditions. PCRs were carried out using a GeneAmp 9700 (Applied Biosytems, Foster City, CA, USA) at a total volume of $15 \mu \mathrm{l}$, containing $15 \mathrm{ng}$ of genomic DNA, $10 \times$ GeneAmp Buffer II, $0.1 \mathrm{~mm}$ dNTP (GeneAmp dNTP MIX), $1.5 \mathrm{~mm} \mathrm{MgCl}_{2}$ (of which exon 12 of $\mathrm{KCNH} 2$ accounted for $0.75 \mathrm{~mm}$ ), $1.5 \mathrm{U}$ of AmpliTaq Gold DNA polymerase (Applied Biosystems) and $0.3 \mu \mathrm{M}$ of each primer set. We purified PCR amplicons with Amicon Ultra Centrifugal Filters (Merck Millipore, Bedford, MA, USA), then used the amplified PCR products directly as templates for sequencing reactions, carried out using the BigDye Terminator v1.1 Cycle Sequencing Kit (Applied Biosystems). The sequencing primers were identical to those used for PCR. Sequencing was done with an ABI 3130-Avant Genetic Analyzer (Applied Biosystems). Data analysis was performed automatically using the Applied Biosystems Data Collection and DNA sequencing analysis software.

\section{PCR-restriction fragment length polymorphism analysis to detect} G643S in LQT1 and K897T in LQT2

To investigate the further reliable polymorphism frequencies of the G643S and K897T in a control Japanese population, we analyzed DNA samples from 281 unrelated Japanese individuals with no medical history of cardiac disease, in addition to 100 Japanese individuals of the control group, using PCRrestriction fragment length polymorphism (RFLP) methods to detect G1927A (a G643S polymorphism on LQT1) or A2690C (a K897T polymorphism on LQT2). PCR products amplified using the same primer set as for PCR of exon 16 on LQT1 gene were digested with PstI enzyme to detect G643S. ${ }^{20}$ Additionally, PCR-RFLP to detect K897T on LQT2 gene was done using the $B s a A I$ enzyme, as described in a previous paper. ${ }^{21}$ We used the Simadzu MCE202 MultiNA (Shimadzu, Kyoto, Japan) to view digested fragments patterns.

\section{RESULTS}

The 10 individuals in the psychotropic drug group were 5 males and 5 females with an average age in the 30 s at the time of death. While no electrocardiograms were available for any individuals, all were free of previous diagnosed cardiac disease. Additionally, two or more psychotropic drugs had been prescribed in for all 10 individuals. Table 3 shows a summary of polymorphisms detected in LQT1 and LQT2. Among the individuals in this group, our analysis identified two missense polymorphisms (P448R and G643S) and two silent polymorphisms in LQT1 and one missense polymorphism (K897T) and five silent polymorphisms in LQT2. In the psychotropic drug group, we identified the $\mathrm{P} 448 \mathrm{R}$ polymorphism in one individual (10\%), the G643S polymorphism in six individuals $(60 \%)$ and the K897T polymorphism in one individual (10\%). In contrast, P448R, G643S and K897T were detected, respectively, in 21, 11 and 6\% of the control group $(n=100)$. Thus, G643S polymorphism frequencies differ significantly between the psychoactive drug group and the control group $(P<0.001)$. For the P448R and the K897T polymorphism, we found no statistically significant difference in frequency between the psychotropic drug group and the control group $(P>0.05)$. Figure 1 describes examples of direct sequencing data for the polymorphism resulting in the missense polymorphism of G643S on LQT1 or K897T on LQT2 that may be associated with potassium channel dysfunction. In samples from the group of 381 unrelated Japanese individuals, the G643S polymorphism on LQT1 or the K897T polymorphism on LQT2 was detected, respectively, in $42(11 \%)$ and $35(9.2 \%)$ individuals. Thus, the results of the polymorphism frequencies in the group of 381 individuals are much the same as those among the control group $(n=100)$. Figure 2 describes the results of the PCR-RFLP methods in the psychotropic

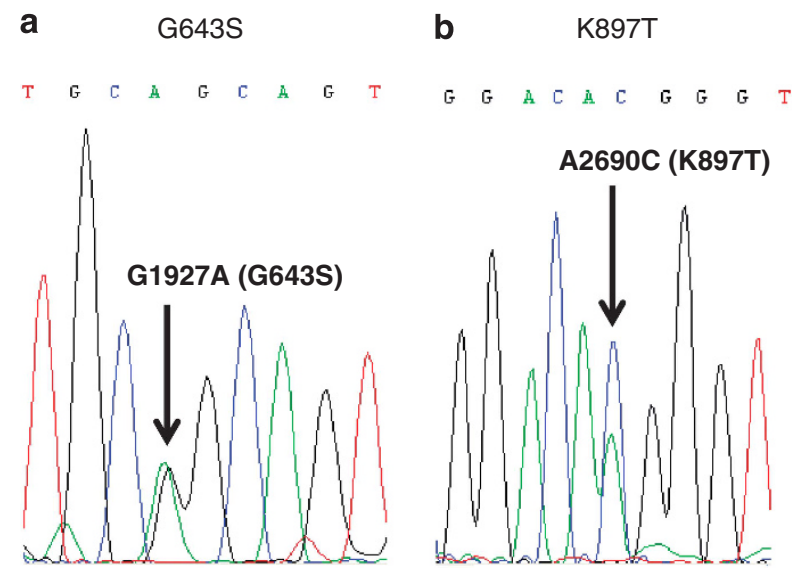

Figure 1 Direct sequencing of the $K C N Q 1$ or $K C N H 2$ gene shows each nucleotide and the amino-acid substitutions. (a) Arrow indicates nucleotide position (G1927A) associated with the G643S mutation; (b) arrow indicates nucleotide position (A2690C) associated with the K897T mutation.

Table 3 Summary of polymorphisms detected in present study

\begin{tabular}{|c|c|c|c|c|c|c|}
\hline Gene & Nucleotide change & Amino-acid change & Position within protein & Exon & $\begin{array}{l}\text { Number detected } \\
\text { in psychotropic } \\
\text { drug group }(\mathrm{n}=10)\end{array}$ & $\begin{array}{l}\text { Allele frequency } \\
\text { in control group } \\
(\mathrm{n}=100)\end{array}$ \\
\hline \multirow[t]{4}{*}{ KCNQ1 (LQT1) } & C435T & $|145|$ & S1-S2 & 2 & 2 (cases 1,9 ) & 0.11 \\
\hline & C1343G & P448R & C-terminal & 10 & 1 (case 1 ) & 0.21 \\
\hline & G1638A & S546S & C-terminal & 13 & $3($ cases $4,6,7)$ & 0.56 \\
\hline & G1927A & G643S & C-terminal & 16 & 6 (cases $1,2,3,4,5,6$ ) & 0.11 \\
\hline \multirow[t]{6}{*}{ KCNH2 (LQT2) } & T1467C & |489| & S2-S3 & 6 & 5 (cases $1,2,6,7,8)$ & 0.41 \\
\hline & T1539C & F513F & S3 & 6 & 5 (cases $1,2,6,7,8)$ & 0.37 \\
\hline & G1692A & L564L & S5 & 7 & 2 (cases 1,8$)$ & 0.03 \\
\hline & C1956T & Y652Y & S6 & 8 & 1 (case 1 ) & 0.08 \\
\hline & A2690C & K897T & C-terminal & 11 & 1 (case 2) & 0.06 \\
\hline & G2732C & G911G & C-terminal & 12 & 3 (cases $7,9,10)$ & 0.27 \\
\hline
\end{tabular}

The nucleotide numbering starts from the ATG start codon. GenBank accession numbers AF000571 (KCNQ1) and AF363636 (KCNH2). 

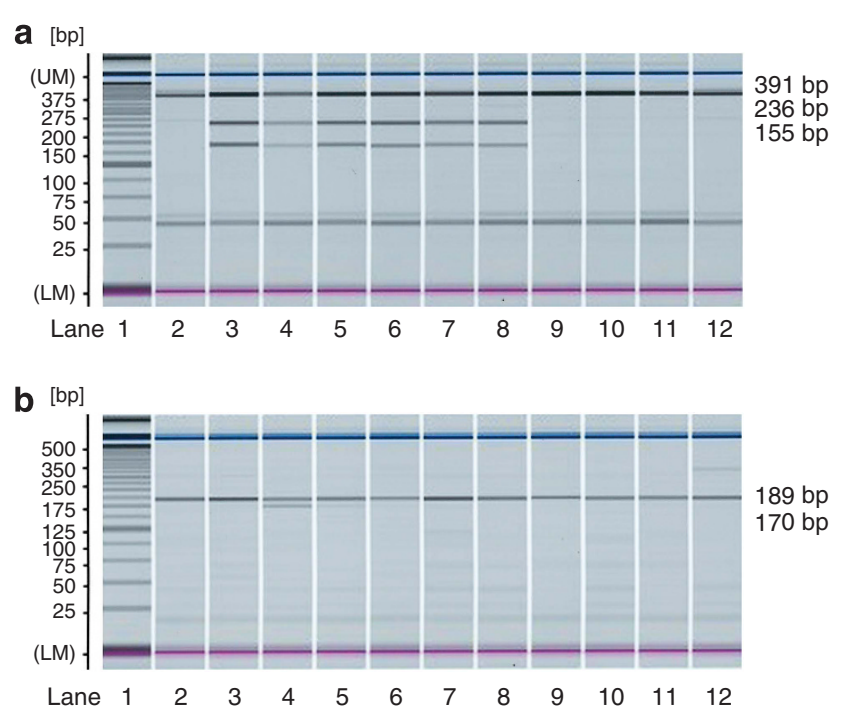

Figure 2 Results obtained with PCR-RFLP analysis for the psychotropic drug group. (a) Result of analysis using Pstl to detect G643S; (b) results of analysis to detect K897T using BsaAl. Lane 1, 25 bp ladder marker; lane 2, mutation-negative sample; lanes 3-12, samples of the psychotropic drug group (cases 1-10).

drug group. Amplicons with the G643S polymorphism were digested with PstI into 236 and 155 bp fragments; amplicons with the K897T polymorphism were digested with BsaAI into 189 and $170 \mathrm{bp}$ fragments.

\section{DISCUSSION}

Unexpected sudden death has been observed in patients with psychological disorders. The mortality rate among psychiatric patients is significantly higher than in the general population. ${ }^{1-3}$ While patient autopsies in many cases can clearly identify cause of death (for example, pulmonary thromboembolism, malignant syndrome and poisoning due to drug overdose), autopsy findings in some cases point to no clear cause of death. In these cases, sudden death of natural causes may have functional origins, including life-threatening arrhythmias. An earlier large epidemiologic cohort study indicated that patients taking psychotropic drugs had higher rates of fatal sudden cardiac death due to life-threatening arrhythmias. ${ }^{7,22}$ Review reports mention described the risk of sudden death in patients taking psychotropic drugs. ${ }^{4,9,23,24}$ LQTS followed by life-threatening arrhythmias can be classified into two types: congenital LQTS and acquired LQTS. The various drugs that cause acquired LQTS are believed to affect the cardiac potassium channels, and psychotropic drugs associated with this risk may also block potassium channels. ${ }^{4-6,10,25-27}$ We hypothesize that asymptomatic individuals carrying certain polymorphisms in LQT genes associated with potassium channel dysfunction may be at risk of life-threatening arrhythmias if exposed to psychotropic drugs. The study reported here entailed genetic screening for two major genes of LQT1 and LQT2 that encode for cardiac potassium channels.

In the psychotropic drug group, we identified three missense polymorphisms in LQT1 and LQT2. While the P448R polymorphism on LQT1 is seemed to have little effect on potassium channel, ${ }^{28}$ the G643S polymorphism on LQT1 and the K897T polymorphism on LQT2 may be associated with potassium channel dysfunction. ${ }^{29-32}$ The G643S polymorphism was detected at an unusually high rate $(60 \%)$ in the psychotropic drug group. An analysis of 381 healthy
Japanese individuals identified 42 (11\%) carrying the G643S missense polymorphism. A previous study had reported a 9\% rate of G643S polymorphism among 110 Japanese individuals. ${ }^{29}$ This reported rate is close to the polymorphism frequency (11\%) among the 381 individuals in our present study. Another previous study found a polymorphism frequency of $6 \%$ among a group of 134 Asians consisting of 60 Japanese, 56 Chinese and 18 Filipino. ${ }^{29}$ The result of our study suggests that the individuals administered psychotropic medication and who die suddenly and unexpectedly of unidentifiable causes carry the G643S polymorphism at an unusually high rate compared with the general Japanese population. A previous functional analysis showed that the G643S polymorphism results in $30 \%$ reductions in the slow delayed rectifier $\mathrm{K}^{+}$current $\left(I_{\mathrm{Ks}}\right){ }^{30}$ The report indicates the G643S polymorphism causing mild $I_{\mathrm{Ks}}$ channel dysfunction might predispose gene carriers to life-threatening arrhythmias in the presence of appropriate precipitating factors, such as hypokalemia or bradyarrhythmia. Thus, asymptomatic individuals carrying the G643S polymorphism in the LQT1 genes associated with mild $I_{\mathrm{Ks}}$ channel dysfunction may face increased risk of life-threatening arrhythmias if exposed to psychotropic drugs that block the potassium channels.

Our analysis of 381 healthy Japanese individuals showed that 35 (or 9.2\%) carried a missense polymorphism of the K897T on LQT2. We detected this polymorphism in one patient in the psychotropic drug group. This polymorphism has been reported to be a common variant in Caucasian populations, with a frequency of $33 \%$, while the frequency among Asians is reported to be $7.5 \%{ }^{29}$ However, some previous papers suggest that the K897T polymorphism may be associated with LQTS. ${ }^{31,32}$ A previous paper based on functional analysis for the polymorphism suggests that the K897T polymorphism results in slightly lower current density than the wild type. ${ }^{32}$ The paper also reported that small decreases in current density attributable to the K897T polymorphism had little potential to cause disease alone but that the presence of the K897T polymorphism could reinforce the effects of the delayed rectifier $\mathrm{K}^{+}\left(I_{\mathrm{kr}}\right)$ reduction caused by certain settings such as QT-prolonging drugs or co-inheritance of an LQTS polymorphism. Thus, asymptomatic individuals carrying the K897T may face increased risk of life-threatening arrhythmias if exposed to psychotropic drugs that block the potassium channels. The one patient in our study carrying the K897T polymorphism also expressed the G643S polymorphism on LQT1, making it difficult to elucidate any relation between the K897T polymorphism and life-threatening arrhythmias among the patients taking psychotropic drugs.

We conclude that administering psychotropic drug therapy to individuals carrying the G643S in the LQT1 may increase the risk of life-threatening arrhythmias due to prolonged QT intervals. Screening for the G643S polymorphism in LQT1 before prescribing psychotropic drugs may reduce the risk of life-threatening arrhythmias. Firm support for this observation will require significantly greater numbers of sudden death cases involving autopsy-negative patients administered medication for psychological disorders. Although greater number of non-sudden death cases taking the drugs and carrying the G643S polymorphism must be analyzed, screenings for the G643S polymorphism among elderly autopsy cases not due to life-threatening arrhythmia $(n=28)$ taking psychotropic drugs that can potentially prolong QT interval indicates 4 of 28 individuals are carriers of the G643S polymorphism (detailed data not shown). Sudden death cases in patients taking psychotropic drugs may require the coexpression of polymorphisms on LQT genes other than LQT1 or LQT2. Analysis of other LQT genes is required to explore this 
possibility. Additionally, no electrocardiograms were available for the sudden death cases examined in this study. Cases of sudden death in which such records are available should be helpful.

\section{CONFLICT OF INTEREST}

The authors declare no conflict of interest.

1 Harris, E. C. \& Barraclough, B. Excess mortality of mental disorder. Br. J. Psychiatry 173, 11-53 (1998)

2 Raedler, T. J. Cardiovascular aspects of antipsychotics. Curr. Opin. Psychiatry 23, 574-581 (2010)

3 Timour, Q., Frassati, D., Descotes, J., Chevalier, P., Christé, G. \& Chahine, M. Sudden death of cardiac origin and psychotropic drugs. Front. Pharmacol. 3, 1-9 (2012)

4 Haddad, P. M. \& Anderson, I. M. Antipsychotic-related QTC prolongation, Torsade de pointes and sudden death. Drugs 62, 1649-1671 (2002).

5 Kongsamut, S., Kang, J., Chen, X. L., Roehr, J. \& Rampe, D. A comparison of the receptor binding and HERG channel affinities for a series of antipsychotic drugs. Eur. J. Pharmacol. 450, 37-41 (2002)

6 Killeen, M. J. Antipsychotic-induced sudden cardiac death: examination of an atypical reaction. Expert Opin. Drug Saf. 8, 249-252 (2009).

7 Ray, W. A., Chung, C. P., Murray, K. T., Hall, K. \& Stein, C. M. Atypical antipsychotic drugs and the risk of sudden cardiac death. N. Engl. J. Med. 360, 225-235 (2009).

8 Ramos-Ríos, R., Arrojo-Romero, M., Paz-Silva, E., Carballal-Calvo, F., Bouzón-Barreiro, J. L., Seoane-Prado, J. et al. QTc interval in a sample of long-term schizophrenia inpatients. Schizophr. Res. 116, 35-43 (2010).

9 Alvarez, P. A. \& Pahissa, J. QT alterations in psychopharmacology: proven candidates and suspects. Curr. Drug Saf. 5, 97-104 (2010).

10 Lazarczyk, M. J., Bhuiyan, Z. A., Perrin, N. \& Giannakopoulos, P. Selective acquired long QT syndrome (saLQTS) upon riperodone treatment. BMC Psychiatry 12, 220 (2012).

11 Raymond, L. A trusted partner providing reliable information on medicines, http:// www.azcert.org/ (25 May 2013).

12 Ozeki, Y., Fujii, K., Kurimoto, N., Yamada, N., Okawa, M., Aoki, T. et al. QTc prolongation and antipsychotic medications in sample of 1017 patients with schizophrenia. Prog. Neuropsychopharmacol. Biol. Psychiatry 34, 401-405 (2010).

13 Mahida, S., Hogarth, A. J., Cowan, C., Tayebjee, M. H., Graham, L. N. \& Pepper, C. B. Genetics of congenital and drug-induced long QT syndromes: currents evidence and future research perspectives. J. Interv. Card. Electrophysiol. 37, 9-19 (2013).

14 Priori, S. G., Napolitano, C. \& Schwartz, P. J. Low penetrance in the long-QT syndrome: clinical impact. Circulation 99, 529-533 (1999).

15 Brugada, R. Sudden death: managing the family, the role of genetics. Heart 97, 676-681 (2011).

16 Splawski, I., Shen, J., Timothy, K. W., Vincent, G. M., Lehmann, M. H. \& Keating, M. T. Genomics structure of three long QT syndrome genes: KVLQT1, HERG, and KCNE1. Genomics 51, 86-97 (1998).
17 Jongbloed, R., Marcelis, C., Velter, C., Doevendans, P., Geraedts, J. \& Smeets, H. DHPLC analysis of potassium ion channel genes in congenital long QT syndrome. Hum. Mutat. 20, 382-391 (2002).

18 Itoh, T., Tanaka, T., Nagai, R., Kamiya, T., Sawayama, T., Nakayama, T. et al. Genomic organization and mutational analysis of HERG, a gene responsible for familial long QT syndrome. Hum. Genet. 102, 435-439 (1998).

19 Hecker, K. H. \& Roux, K. H. High and low annealing temperatures increase of both specificity and yield in touchdown and stepdown PCR. Biotechniques 20,478-485 (1996).

20 Ozawa, T., Ito, M., Tamaki, S., Yao, T., Ashihara, T., Kita, Y. et al. Gender and age effects on ventricular repolarization abnormality in Japanese general carriers of a G643S common single nucleotide polymorphism for the KCNQ1 gene. Circ. J. 70, 645-650 (2006).

21 Bezzina, C. R., Verkerk, A. O, Busjahn, A., Jeron, A., Erdmann, J., Koopmann, T. T. et al. A common polymorphism in KCNH2(HERG) hastens cardiac repolarization. Cardiovasc. Res. 59, 27-36 (2003).

22 Hennessy, S., Bilker, W. B., Knauss, J. S., Margolis, D. J., Kimmel, S. E., Reynolds, R. F. et al. Cardiac arrest and ventricular arrhythmia in patients taking antipsychotic drugs: cohort study using administrative data. BMJ 325, 1070 (2002).

23 Ray, W. A., Meredith, S., Thapa, P. B., Meador, K.G., Hall, K. \& Murray, K. T. Antipsychotics and the risk of sudden cardiac death. Arch. Gen. Psychiatry 58, 1161-1167 (2001).

24 Justo, D., Prokhorov, V., Heller, K. \& Zeltser, D. Torsade de pointes induced by psychotropic drugs and the prevalence of its risk factors. Acta. Psychiatr. Scand. 111, 171-176 (2005)

25 Paulussen, A. D., Gilissen, R. A., Armstrong, M., Doevendans, P.A., Verhasselt, P., Smeets, $\mathrm{H}$. J et al. Genetic variations of KCNQ1, KCNH2, SCN5A, KCNE1, and KCNE2 in drug-induced long QT syndrome patients. J. Mol. Med. 82, 182-188 (2004).

26 Roden, D. M. \& Viswanathan, P. C. Genetics of acquired long QT syndrome. J. Clin. Invest. 115, 2025-2032 (2005).

27 Michelsen, J.W. \& Meyer, J.M. Cardiovascular effects of antipsychotics. Expert Rev. Neurother. 7, 829-839 (2007).

28 Sharma, D., Glatter, K. A., Timofeyev, V., Tuteja, D., Zhang, Z., Rodriguez, J. et al. Characterization of a KCNQ1/KVLQT1 polymorphism in Asia families with LQT2: implications for genetic testing. J. Mol. Cell. Cardiol. 37, 79-89 (2004).

29 Ackerman, M. J., Tester, D. J, Jones, G. S, Will, M. L., Burrow, C. R \& Curran, M. E. Ethnic differences in cardiac potassium channel variants: implications for genetic susceptibility to sudden cardiac death and genetic testing for congenital long QT syndrome. Mayo Clin. Proc. 78, 1479-1487 (2003).

30 Kubota, T., Horie, M., Takano, M., Yoshida, H., Takenaka, K., Watanabe, E. et al. Evidence for a single nucleotide polymorphism in the KCNQ1 potassium channel that underlies susceptibility to life-threatening arrhythmias. J. Cardiovasc. Electrophysiol. 12, 1223-1229 (2001).

31 Pietilä, E., Fodstad, H. Niskasaari, E., Laitinen, P. PJ., Swan, $H$, Savolainen, M et al. Association between HERG K897T polymorphism and QT interval in middle-age Finnish women. J. Am. Cell Cardiol. 40, 511-514 (2002).

32 Crotti, L., Lundquist, A. L., Insolia, R., Pedrazzini, M., Ferrandi, C., De Ferrari, G. M. et al. KCNH2-K897T is a genetic modifier of latent congenital long-QT syndrome. Circulation 112, 1251-1258 (2005) 